\title{
Correlación del Finnish Diabetes Risk Score y la Hemoglobina Glucosilada en la Identificación de Diabetes y Prediabetes en Docentes Universitarios de Ciencias de la Salud.
}

\section{Correlation of the Finnish Diabetes Risk Score and Glycosylated Hemoglobin for Screening of Undiagnosed Diabetes and Prediabetes in Professors of Health Sciences.}

\author{
Nayali López-Balderas ${ }^{1}$, Beatriz González-Jiménez ${ }^{2}$, Flor Patricia Rosas Murga ${ }^{2}$, Emma Delfina López \\ Ramos $^{2}$, Rosa María Torres-Hernández ${ }^{2} \mathrm{P}$ \\ ${ }^{1}$ Instituto de Medicina Forense, Universidad Veracruzana, Boca del Río, México; \\ 2 UV-CA-477 "Investigación Clínica" Facultad de Medicina, Universidad Veracruzana, Veracruz, México. \\ *Dirigir correspondencia a: rotorres@uv.mx
}

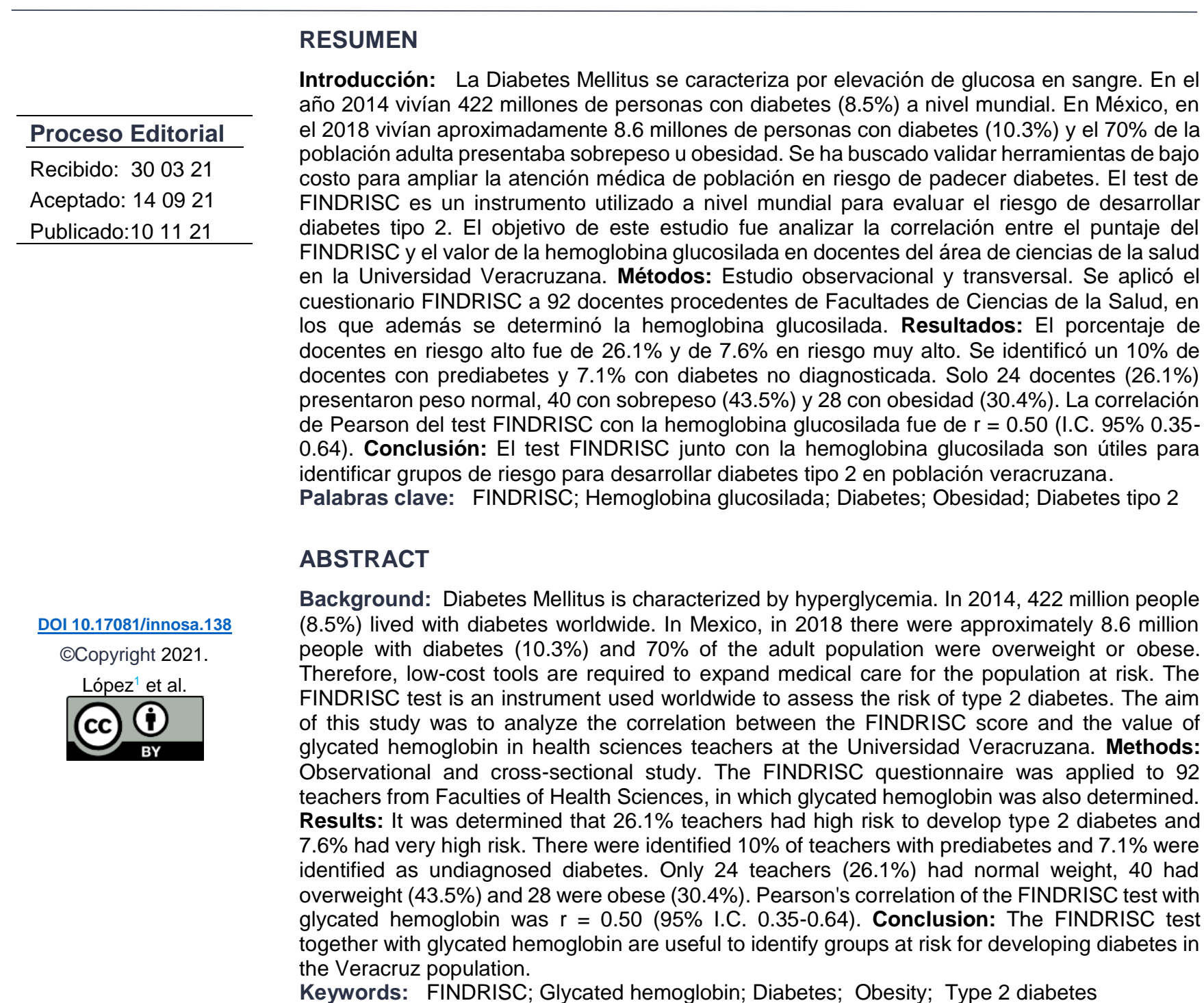

Cómo citar: López-Balderas N, González B, Rosas F, López E, Torres R. Correlación del Finnish Diabetes Risk Score y la Hemoglobina Glucosilada en la Identificación de Diabetes y Prediabetes en Docentes Universitarios de Ciencias de la Salud. Ciencia e Innovación en Salud. 2021. e137: 289-297 DOI 10.17081/innosa.138 


\section{INTRODUCCIÓN}

La Diabetes Mellitus es una enfermedad metabólica crónica que se caracteriza por la elevación de glucosa en sangre. Se puede clasificar en 4 categorías: el tipo 1, originada por un proceso autoinmune que destruye las células $\beta$ creando una deficiencia de insulina; el tipo 2 presenta una pérdida progresiva de la secreción de insulina, o bien, resistencia absoluta o relativa a la acción de la insulina en tejidos periféricos, la tercera es la diabetes gestacional y finalmente la originada por otras causas. De estas, la más frecuente a nivel mundial es el tipo $2(1,2)$.

El aumento de los casos de diabetes tipo 2 se ha convertido en un problema de salud publica. Según la Organización Mundial de la Salud (OMS), el número de personas con diabetes aumentó de 108 millones en 1980 a 422 millones en 2014 (prevalencia en mayores de 18 años de $4,7 \%$ a $8,5 \%$, respectivamente). Esta enfermedad impacta en los gastos en salud, no solo por el número de muertes directas (1.6 millones de muertes en el año 2016), sino también por las comorbilidades que afectan a la población económicamente activa, ya que las principales complicaciones son la insuficiencia renal, ceguera, amputación de miembros inferiores y el mayor riesgo cardiovascular $(\underline{1}, \underline{3})$. La Federación Internacional de Diabetes (FID) ha estimado que para el año 2030 habrá alrededor de 578 millones de adultos viviendo con diabetes, aproximadamente el $10.2 \%$ de la población mundial (4).

México se encuentra entre los países con mayor prevalencia $(>12 \%)$ a nivel mundial (4). Según la Encuesta Nacional de Salud (ENSANUT), en el año 2018 el estado de Veracruz tiene una prevalencia de entre $10.8-11.9 \%$ ( $\underline{5})$.

El diagnóstico de la diabetes se basa en determinar la concentración de glucosa en plasma empleando alguna de las siguientes pruebas: la glucosa plasmática en ayunas, concentración de glucosa a las 2 horas después de una prueba de tolerancia oral a la glucosa $(75 \mathrm{~g})$, y la hemoglobina glucosilada (HbA1c o AC1). A partir de estas, se ha logrado identificar un estado de "prediabetes", en el cual los niveles de glucosa son más altos que los parámetros normales, pero menores a los criterios de diabetes, estas personas tienen alto riesgo de desarrollar diabetes en los siguientes 5 a 10 años (1,2). Adicionalmente, se ha estimado que casi el $50 \%$ de los individuos mayores de 20 años con diabetes no han sido diagnosticados por falta de prevención y acceso a las pruebas $(\underline{2}, \underline{4})$.

Por tal motivo, para ampliar y mejorar la atención temprana de la diabetes, se sugiere utilizar herramientas no invasivas y de bajo costo para identificar a los individuos en riesgo (prediabetes) o no diagnosticados $(2,4)$. El test de FINDRISC (Finnish Diabetes Risk Score) es un cuestionario que se utiliza como prueba de cribado, a través de su puntuación permite identificar aquellas personas con mayor riesgo de desarrollar diabetes tipo 2 y que son candidatos a una evaluación con pruebas de laboratorio $(\underline{3}, \underline{6}, \underline{7})$.

En México existen pocos estudios de validación del FINDRISC en población no diagnosticada, en los cuales se han reportado prevalencias de individuos en prediabetes de entre $23-39 \%$, utilizando la glucosa en ayunas o la HbA1c como criterio de confirmación. En general, estos reportes concluyen que el cuestionario es una herramienta útil para el tamizaje en población mexicana, hasta con un $80 \%$ de sensibilidad ( $\underline{8}, \underline{9}-\underline{12})$. 
El objetivo de este estudio fue analizar la correlación entre el puntaje del cuestionario FINDRISC y el valor de la hemoglobina glucosilada como herramientas para la detección de prediabetes y diabetes en adultos que laboran como docentes del área de ciencias de la salud en la Universidad Veracruzana.

\section{MÉTODOS}

Estudio transversal descriptivo, realizado durante el periodo de enero a julio de 2018. El tamaño de muestra se calculo con base en la fórmula de muestras finitas, con un nivel de confianza 95\%, resultando en un tamaño de muestras de 117 individuos. El reclutamiento se realizó mediante un muestreo no probabilístico por conveniencia. Un total de 92 docentes sin diagnóstico de diabetes que laboraban durante el semestre en curso, en las distintas facultades del área de ciencias de la Salud de la región Veracruz de la Universidad Veracruzana: Medicina, Nutrición, Psicología, Educación Física, Enfermería, Bioanálisis y Odontología, aceptaron participar después de recibir la información y firmar el consentimiento informado.

2.1. Evaluación clínica de los participantes. A cada participante se les aplicó el cuestionario FINDRISC (Finnish Diabetes Risk Score), el cual evalúa edad, índice de masa corporal (IMC), perímetro abdominal, tratamiento antihipertensivo, frecuencia del consumo de frutas y verduras, actividad física, antecedentes de glucemia elevada y antecedente familiar de diabetes. Cada respuesta confiere una puntuación, el puntaje final se puede interpretar en las siguientes categorías: Riesgo bajo (<7 puntos), ligeramente elevado, (7-11 puntos), moderado (11- 14 puntos), alto (15-20 puntos), y muy alto (>20), asociado al riesgo de desarrollar diabetes en los próximos 10 años del 1\%, 4\%, 17\%, 33\% y 50\% respectivamente (ㅁ, $\underline{7})$.

Para calcular IMC se midió peso y talla con bascula digital y estadiómetro clásico. Según los criterios de la OMS, se consideró sobrepeso un IMC igual o superior a 25, y obesidad como un IMC igual o superior a 30. Se midió el perímetro abdominal localizando el punto medio entre la última costilla y la espina iliaca.

2.2. Estudios de laboratorio. A los docentes que aceptaron participar, se les citó en un consultorio de la Facultad de Medicina Región Veracruz, en donde se les tomó una muestra de $3 \mathrm{ml}$ sangre en tubo con EDTA para la determinación cuantitativa de hemoglobina glucosilada. Un total 70 docentes (de los 92 que respondieron el cuestionario) acudieron al consultorio. Las muestras se procesaron según las indicaciones del proveedor (Spinreact en analizador automático de química sanguínea HLAB H-100). Según la recomendación de la Asociación Americana de Diabetes se clasificaron como prediabetes aquellos que presentaron valor de $\mathrm{HbA} 1 \mathrm{c}$ de entre $5.4-6.4 \%$ y diabetes $\geq 6.5 \%$ (2) .

2.3. Análisis estadístico. El registró de datos, la estadística descriptiva, prueba ANOVA, Chicuadrada y análisis de correlación de Pearson se realizó con el software IBM SPSSStatistics versión 22. Se obtuvieron frecuencias absolutas y relativas, la media, y la estadística inferencial con un intervalo de confianza del $95 \%$.

2.4. Aspectos éticos. Los procedimientos se llevaron a cabo de acuerdo con la normatividad internacional (Declaración de Helsinki) y nacional sobre investigación en seres humanos. El 
protocolo fue aprobado por el comité de investigación y de ética en investigación de la Facultad de Medicina Región Veracruz.

\section{RESULTADOS}

Se incluyeron 92 docentes de la Universidad Veracruzana Región Veracruz, 11 pertenecientes a la Facultad de Nutrición (12\%), 47 de la Facultad de Medicina (51.1\%), 15 de Educación Física (16.3\%), 10 de Psicología (10.9\%), y 9 de otras facultades (9.8\%). El 48.9\% corresponden a docentes del sexo masculino. La edad promedio fue de $48.4 \pm 15.8$ años. El IMC promedio fue de $28.3 \pm 5.5 \mathrm{Kg} / \mathrm{m} 2$, encontrándose con peso normal a 24 docentes $(26.1 \%)$, 40 con sobrepeso (43.5\%) y 28 con obesidad (30.4\%) (Tabla 1$)$.

Se encontraron 23 docentes con tratamiento antihipertensivo (25\%) y solo $14(15.2 \%)$ reportaron antecedentes de glucemia elevada. Una tercera parte, 26 docentes (28.3\%) manifestaron no tener antecedentes familiares de diabetes, 43 (46.7\%) tiene antecedentes de familiares de primer grado. Solo $34(37 \%)$ realizan alguna actividad física (Tabla 1).

A partir de las puntuaciones obtenidas del cuestionario FINDRISC, se encontró que 17 (18.5\%) docentes tuvieron un riesgo bajo (<7 puntos), 28 (30.4\%) con riesgo ligeramente elevado (711 puntos), 16 (17.4\%) con riesgo moderado (12-14 puntos), 24 (26.1\%) con riesgo alto (1520 puntos) y $7(7.6 \%)$ con riesgo muy alto (>20 puntos) (Tabla 1).

En las 70 muestras obtenidas para el análisis de $\mathrm{HbA1c}$, se encontró que un $82.9 \%$ de los docentes (58) presentaron valores en el rango normal, 10\% en un rango de prediabetes y $7.1 \%$ en el rango considerado como diabetes (Tabla 1).

Al analizar el riesgo según el FINDRISC, entre las distintas facultades de ciencias de la salud, se observó que la facultad de nutrición fue la única que no tuvo población con riesgo alto y muy alto (mayor o igual a 15) el resto tuvo entre $31-50 \%$ de docentes con esos riesgos. Sin embargo, no se obtuvo una diferencia estadísticamente significativa $(p>0.05)$. Todas las facultades tuvieron altos porcentajes de sobrepeso y obesidad (entre 65 y $90 \%$ ). Según los valores de HbA1c, la facultad de nutrición y los agrupados como otros, presentaron el valor promedio más bajo (4.62 y $4.5 \%$ ) y no tuvieron casos clasificados como diabetes y prediabetes, mientras que el resto tuvo entre $18-50 \%$, en estos parámetros si hubo diferencias estadísticamente significativas ( $p=0.002$ y 0.017 , respectivamente) (Tabla 2).

El análisis de correlación del cuestionario FINDRISC con los valores de la hemoglobina glucosilada mediante la correlación de Pearson, resulto en un valor de $r=0.50$ (I.C. 95\% 0.350.64) con significancia de $\mathrm{p}<0.001$ (Figura 1). Lo cual representa una correlación positiva moderada pero estadísticamente significativa, entre el puntaje del cuestionario FINDRISC y el valor de la hemoglobina glucosilada (HbA1c). 
Tabla 1. Datos obtenidos del cuestionario FINDRISC aplicado a docentes de Ciencias de la Salud de la Universidad Veracruzana Región Veracruz.

\begin{tabular}{|c|c|c|c|}
\hline VARIABLE & $\begin{array}{l}\text { FRECUENCIA } \\
\text { ABSOLUTA }\end{array}$ & $\begin{array}{l}\text { FRECUENCIA } \\
\text { RELATIVA }\end{array}$ & \\
\hline \multirow[t]{2}{*}{ Genero } & Masculino & 45 & $48.9 \%$ \\
\hline & Femenino & 47 & $51.1 \%$ \\
\hline \multirow[t]{3}{*}{ IMC } & Normal & 24 & $26.1 \%$ \\
\hline & Sobrepeso & 40 & $43.5 \%$ \\
\hline & Obesidad & 28 & $30.4 \%$ \\
\hline \multirow[t]{2}{*}{ Actividad física } & No & 58 & $63 \%$ \\
\hline & SI & 34 & $37 \%$ \\
\hline \multirow{2}{*}{$\begin{array}{l}\text { Tratamiento } \\
\text { antihipertensivo }\end{array}$} & No & 69 & $75 \%$ \\
\hline & $\mathrm{Si}$ & 23 & $25 \%$ \\
\hline \multirow{2}{*}{$\begin{array}{l}\text { Antecedentes } \\
\text { de glucemia } \\
\text { elevada }\end{array}$} & No & 78 & $84.8 \%$ \\
\hline & $\mathrm{Si}$ & 14 & $15.2 \%$ \\
\hline \multirow{3}{*}{$\begin{array}{l}\text { Antecedentes } \\
\text { familiares de } \\
\text { DM }\end{array}$} & No & 26 & $28.3 \%$ \\
\hline & $\begin{array}{l}\text { Familiares de } \\
\text { primer grado }\end{array}$ & 43 & $46.7 \%$ \\
\hline & $\begin{array}{l}\text { Familiares de } \\
\text { segundo } \\
\text { grado }\end{array}$ & 23 & $25 \%$ \\
\hline \multirow{5}{*}{$\begin{array}{l}\text { FINDRISC } \\
\text { Puntos } \\
\text { Corte }\end{array}$} & $\begin{array}{ll}<7 & \text { Riesgo } \\
\text { baio } & \end{array}$ & 17 & $18.5 \%$ \\
\hline & $\begin{array}{l}7-11 \quad \text { Riesgo } \\
\text { ligeramente } \\
\text { elevado }\end{array}$ & 28 & $30.4 \%$ \\
\hline & $\begin{array}{l}\text { 12-14 Riesgo } \\
\text { moderado }\end{array}$ & 16 & $17.4 \%$ \\
\hline & $\begin{array}{l}15-20 \text { Riesgo } \\
\text { alto }\end{array}$ & 24 & $26.1 \%$ \\
\hline & $\begin{array}{l}>20 \quad \text { Riesgo } \\
\text { muy alto }\end{array}$ & 7 & $7.6 \%$ \\
\hline \multirow{3}{*}{$\begin{array}{l}\mathrm{HbA1c} \\
(\mathrm{n}=70)\end{array}$} & Normal & 58 & $82.9 \%$ \\
\hline & Prediabetes & 7 & $10 \%$ \\
\hline & Diabetes & 5 & $7.1 \%$ \\
\hline
\end{tabular}

Fuente:Elaboración propia

\section{DISCUSIÓN}

La diabetes y las complicaciones que provoca aumentan el riesgo de morir prematuramente y además impactan económicamente en las familias y los sistemas de salud. Por lo cual, es necesario contar con políticas y prácticas de prevención, así como contar con herramientas de diagnóstico temprano que permitan reducir el número de casos y también mejorar la calidad de vida de los pacientes que ya presentan diabetes (1). 
Para ampliar y mejorar la atención temprana de la diabetes, se ha sugerido utilizar herramientas de bajo costo, como los cuestionarios que recopilan información de fácil acceso con la finalidad de identificar población en riesgo, y hacer cambios en el estilo de vida y seguimiento médico. Sin embargo, estos cuestionarios requieren validarse en diferentes poblaciones y con pruebas de laboratorio antes de generalizar su uso. EI FINDRISC es uno de los cuestionarios más utilizados, pero tiene una sensibilidad variable dependiendo de las pruebas utilizadas para confirmarlo o darle seguimiento $(\underline{8}, \underline{13})$.

Tabla 2. Puntaje de riesgo del cuestionario FINDRISK y su validación con HbA1c según la institución de adscripción de los docentes.

\begin{tabular}{|c|c|c|c|c|c|c|}
\hline & Nutrición & Medicina & $\begin{array}{l}\text { Educación } \\
\text { Física }\end{array}$ & Psicología & Otros & $\mathbf{p}$ \\
\hline \multicolumn{7}{|l|}{ FINDRISC } \\
\hline$<7$ & 2 (18.2) & $6(12.8)$ & $3(20.0)$ & $4(40)$ & $2(22.2)$ & 0.12 \\
\hline $7-11$ & 8 (72.7\%) & $13(27.7)$ & $5(33.4)$ & $1(10)$ & $1(11.1)$ & \\
\hline $12-14$ & $1(9.1)$ & $10(21.3)$ & $2(13.3)$ & $1(10)$ & 2 (22.2) & \\
\hline $15-20$ & - & $16(34.0)$ & $3(20.0)$ & $2(20)$ & $3(33.4)$ & \\
\hline$>20$ & - & $2(4.3)$ & $2(13.3)$ & $2(20)$ & $1(11.1)$ & \\
\hline \multicolumn{7}{|l|}{ IMC } \\
\hline Normal & $2(18.2)$ & $16(34.0)$ & - & $2(20)$ & $4(44.4)$ & \\
\hline Sobrepeso & $6(54.5)$ & $19(40.4)$ & 8 (53.3) & $6(60)$ & $1(11.2)$ & 0.12 \\
\hline Obesidad & $3(27.3)$ & $12(25.5)$ & 7 (46.7) & $2(20)$ & $4(44.4)$ & \\
\hline \multicolumn{7}{|l|}{ HbA1C } \\
\hline $\begin{array}{l}\overline{\mathbf{x}} \pm \mathrm{DE}^{\star} \\
\text { Normal }\end{array}$ & $\begin{array}{c}4.62 \pm 0.6 \\
8(100)\end{array}$ & $\begin{array}{c}5.2 \pm 0.7 \\
27(81.8)\end{array}$ & $\begin{array}{l}5.3 \pm 0.7 \\
11(80)\end{array}$ & $\begin{array}{c}6.01 \pm 1.1 \\
3(50)\end{array}$ & $\begin{array}{l}4.5 \pm 0.6 \\
9(100)\end{array}$ & 0.002 \\
\hline Prediabetes & - & $5(15.2)$ & $1(6.7)$ & $1(16.7)$ & - & 0.11 \\
\hline Diabetes & - & $1(3.0)$ & $2(13.3)$ & 2 (33.3) & - & \\
\hline
\end{tabular}

${ }^{*} \overline{\mathrm{X}}=$ promedio, $\mathrm{DE}=$ Desviación estándar.

Fuente:Elaboración propia. 


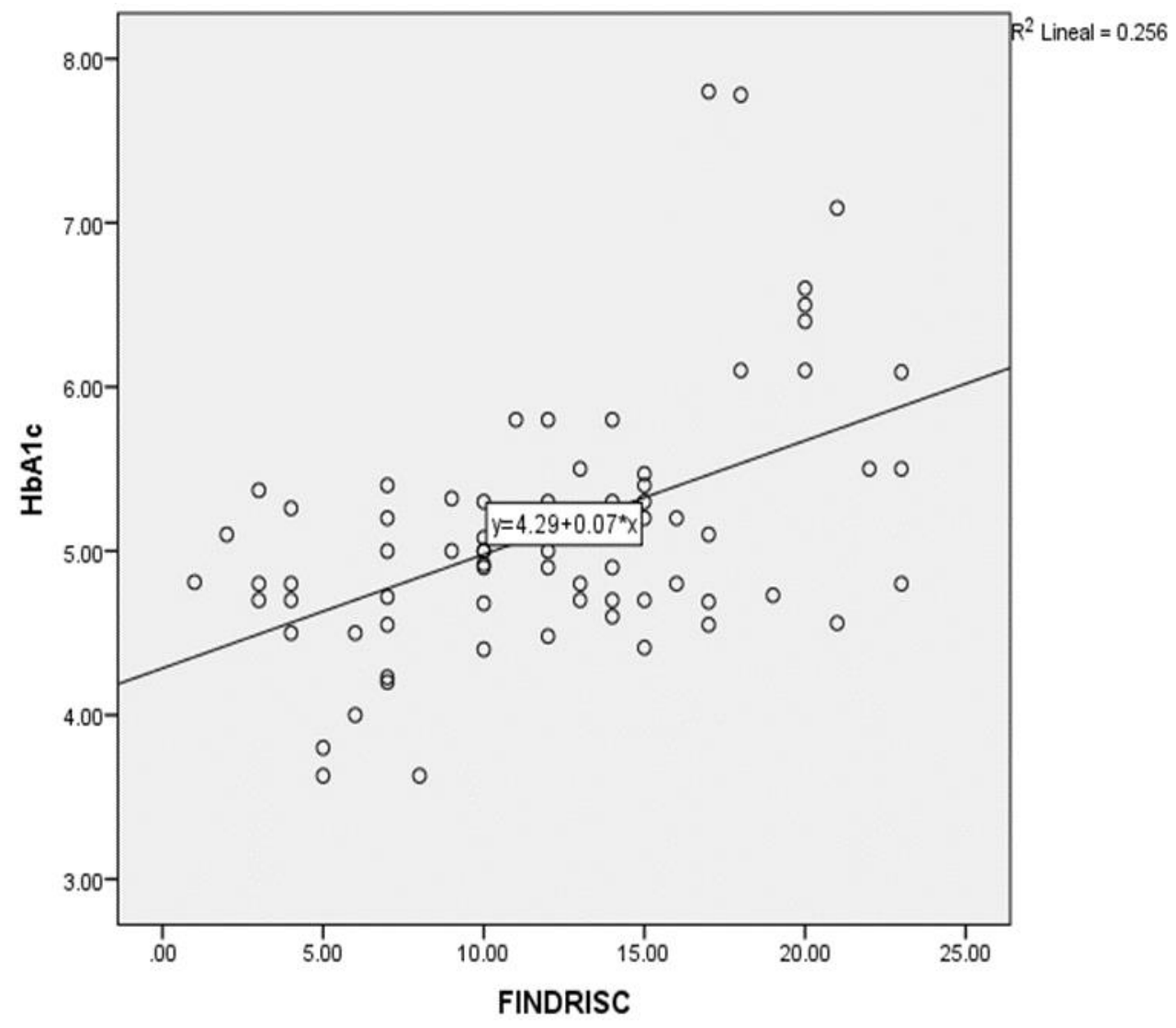

Figura 1. Análisis de correlación de Pearson entre el puntaje obtenido del cuestionario FINDRISC y el valor de hemoglobina glucosilada (HbA1C). Se obtuvo un valor de $r=0.50$ (I.C. 95\% 0.35-0.64) con significancia de $p<$ 0.001 .

Fuente:Elaboración propia.

En este estudio se utilizó el cuestionario FINDRISC y se validó el estado de prediabetes y diabetes no diagnosticada por medio de la hemoglobina glucosilada (HbA1c). Las prevalencias encontradas fueron $10 \%$ y $7.1 \%$, respectivamente, y fueron más bajas que las encontradas en estudios que también utilizaron $\mathrm{HbA1c}$, lo cuales reportan prevalencias de entre $39-44 \%$ de prediabetes y $9-18 \%$ de diabetes no diagnosticada (12-14).

El porcentaje de población en riesgo según el puntaje FINDRISC (26.1\% en riesgo alto y $7.6 \%$ en riesgo muy alto), también fue mas bajo que lo reportado en otras poblaciones mexicanas (mayores al 50\%) (9-12). Adicionalmente, se encontró que el $73.9 \%$ de los docentes presentó obesidad o sobrepeso ( $30.4 \%$ y $43.5 \%$, respectivamente), lo cual es similar al reporte nacional $(76.6 \%)(15)$ y es uno de los principales factores de riesgo reportados en población con prediabetes o diabetes no diagnosticada $(\underline{9}, \underline{11}, \underline{14})$.

Al analizar las distintas facultades, se observó que sólo nutrición y los que fueron agrupados como otros, no tenían población en riesgo alto o muy alto (puntaje mayor o igual a 15) lo cual se correlacionó también con el promedio de $\mathrm{HbA1c}$ y que tampoco tienen casos de prediabetes 
o diabetes. La facultad de medicina y psicología tuvieron los porcentajes más altos en prediabetes y diabetes no diagnosticada, respectivamente. Esto podría estar relacionado con el tipo de alimentación, ya que se ha reportado que la promoción de un régimen alimentario sano y actividad física son esenciales para disminuir el sobrepeso y obesidad, y no sólo los casos de diabetes tipo 2 si no también sus complicaciones (1). Adicionalmente, un estudio reportó que enfermeras de cuidados intensivos tenían menor riesgo que aquellas que no tenían contacto con pacientes 0 atendían pacientes ambulatorios (11), es decir, las actividades que se realizan de manera rutinaria también están relacionadas con un menor riesgo de desarrollar diabetes $(\underline{1}, \underline{15})$.

$\mathrm{La} \mathrm{HbA1c}$ es una de las tres pruebas recomendadas para el diagnóstico de diabetes o prediabetes $(1,2)$ y también es una de las de mayor disponibilidad en los sistemas de salud de México, junto con la glucosa en ayuno (9). En este estudio, se encontró un coeficiente de correlación de Pearson que indica una correlación positiva significativa de $r=0.50$ del cuestionario FINDRISC y la HgAC1. Por lo tanto, es una prueba de laboratorio adecuada para identificar adultos en riesgo o con diabetes no diagnosticada (11-14).

\section{CONCLUSIONES}

Las prevalencias en México de diabetes tipo 2, sobrepeso, obesidad e hipertensión, así como limitaciones al acceso a la atención médica, hacen indispensable contar con herramientas de bajo costo que permitan identificar poblaciones de riesgo y realizar intervenciones tempranas en el estilo de vida para prevenir los casos nuevos y las complicaciones de la diabetes tipo 2. El cuestionario FINDRISC, es una herramienta no invasiva, fácil de aplicar y de bajo costo que junto con la HbA1c mostró en la población veracruzana una correlación positiva y adecuada para la detección y tratamiento oportuno de población en riesgo, pero sobre todo en individuos con riesgo alto o muy alto (puntaje mayor o igual a 15). Así mismo, es una buena herramienta educativa para practicar y concientizar a los estudiantes de medicina sobre opciones de tamizaje de bajo costo en la atención primaria.

Contribución de los autores: Conceptualización, RMTH, BGJ, EDLR y FPRM.; metodología, RMTH, EDLR, NLB y FPRM.; software, NLB, EDLR y FPRM.; validación, BGJ, EDLR y FPRM.; análisis formal, RMTH, NLB, BGJ, EDLR y FPRM.; investigación, EDLR y FPRM.; recursos, RMTH; curación de datos, EDLR y FPRM.; escritura: preparación del borrador original, NLB, EDLR y FPRM.; escritura: revisión y edición, NLB, BGJ.; visualización, RMTH y NLB.; supervisión, RMTH.; administración del proyecto, RMTH y BGJ.; adquisición de fondos, RMTH y BGJ. Todos los autores han leído y aceptado la versión publicada del manuscrito.

Fondos: "Esta investigación no recibió fondos externos". La Facultad de Medicina Región Veracruz de la Universidad Veracruzana, financió este proyecto.

Conflictos de intereses: "Los autores declaran no tener ningún conflicto de intereses".

\section{REFERENCIAS}

1. Informe Mundial sobre la Diabetes 2016, Organización Mundial de la Salud. Disponible en: https://apps.who.int/iris/bitstream/handle/10665/254649/9789243565255spa.pdf;jsessionid=ABDBD503EFE1B1447B46BCB1D8AB905C?sequence =1 [ 
2. American Diabetes Association. 2. Classification and diagnosis of diabetes: Standards of Medical Care in Diabetes 2020. Diabetes Care 2020;43(Suppl. 1):S14-S31. DOI: 10.2337/dc20S002

3. Standl E, Khunti K, Hansen T y Schnell O. The global epidemics of diabetes in the 21st century: Current situation and perspectives. European Journal of Preventive Cardiology 2019; 26(2S): 714. DOI: $10.1177 / 2047487319881021$

4. Atlas de la Diabetes de la Federación Internacional de Diabetes 2019, 9 Ed. Disponible en: https://www.diabetesatlas.org/upload/resources/material/20200302 133352 2406-IDF-ATLASSPAN-BOOK.pdf

5. Encuesta Nacional de Salud y Nutrición (ENSANUT) 2018. Secretaría de Salud, Instituto de Salud Pública e Instituto Nacional de Estadistica y Geografía. Disponible en: https://ensanut.insp.mx/encuestas/ensanut2018/doctos/informes/ensanut 2018 presentacion resultados.pdf

6. Lindstrom J y Toumilehto J. The diabetes Risk Score. Diabetes Care 2003; 26:725731. DOI: $10.2337 /$ diacare.26.3.725

7. Escala FINDRISC La prevención: el mejor tratamiento. Disponible en: http://www.fundacionastrazeneca.es/wp-content/uploads/2020/03/Escala-FindRisc.pdf

8. Vizzuett-Montoya A, López-García M. Type 2 DM and the use of the FINDRISC test for its screening in occupational health in Mexico. Revista Colombiana de Salud Ocupacional 2020; 10(1): e-6419.

9. Leyva R, López-Carrera M, Guzmán E, y Cervantes D. Identificación de prediabetes en grupos de riesgo: una oportunidad para prevenir la enfermedad. Atención Primaria, 2019, 51(10):663664. DOl: 10.1016/j.aprim.2019.03.008

10. Mendiola-Pastrana I, Urbina-Aranda I, Muñoz-Simón A, Juanico-Morales G, López-Ortiz G. Evaluación del desempeño del Finnish Diabetes Risk Score findrisc como prueba de tamizaje para diabetes mellitus tipo 2. Aten Fam. 2018;25(1):22-26. https://www.medigraphic.com/cgibin/new/resumen.cgi?IDARTICULO=76209

11. Sánchez-Jiménez B, Chico-Barba G, Rodríguez-Ventura A, Sámano R, Veruete-Bedolla $D$, Morales-Hernández R. Risk of development of type 2 diabetes in nurses and its relationship with metabolic alterations.Rev. Latino-Am. Enfermagem. 2019;27:e3161. https://www.scielo.br/j/rlae/a/SRXbzsWXTSJwqskvJYTf7PH/?format=pdf\&lang=es

12. González Pedraza A, Ponce E, Toro F, Acevedo O, Dávila R. Cuestionario FINDRISC FINnish Diabetes Risk Score para la detección de diabetes no diagnosticada y prediabetes. Archivos en Medicina Familiar. 2018, 20(1):5-13. https://www.medigraphic.com/pdfs/medfam/amf2018/amf181b.pdf

13. Martínez-Millana A, Argente-Pla M, Valdivieso B, Traver V y Merino-Torres J. Driving Type 2 Diabetes Risk Scores into Clinical Practice: Performance Analysis in Hospital Settings. J. Clin. Med. 2019, 8, 107. https://www.mdpi.com/2077-0383/8/1/107/htm

14. Kumar A, Wong R, Ottenbacher K, y Snih S. Prediabetes, undiagnosed diabetes, and diabetes among Mexican adults: findings from the Mexican Health and Aging Study.Ann Epidemiol. 2016, 26(3):163-170.

15. Rivera J, Colchero A, Fuentes M, González T, Aguilar C, Hernández G y Barquera S. La obesidad en México. Estado de la política pública y recomendaciones para su prevención y control. Instituto Nacional de Salud Publica, 2018, Primera Edición. https://www.insp.mx/produccion-editorial/novedades-editoriales/4971-obesidad-mexicopolitica-publica-prevencion-control.html 